\title{
Opioid Free Anesthesia to Prevent Post Operative Nausea/Vomiting
}

Presenters: Aleksander Acred, Lindsey Blake \& Milena Devineni

\section{Faculty Advisor: Dr. Cindi Dabney}

COLLEGE OF NURSING College of Nursing - The University of Tennessee Health Science Center - Memphis, TN

\section{Purpose}

The purpose of this DNP project is to compare the incidence of postoperative nausea and vomiting (PONV) in opioid-utilizing and opioidfree general anesthesia.

\section{Specific Goals}

-Compare incidence of PONV in opioid-utilizing and opioid-free anesthesia (OFA)

-Compare the need for rescue antiemetics in the immediate postoperative period (up to 24 hours postop) between OFA and non-OFA

-Compare the incidence of breakthrough pain in the immediate postoperative period in OFA and non-OFA

-Determine if OFA provides adequate pain control and prevention of PONV in general anesthesia patients.

\section{Background}

PONV is an extremely common, dangerous side effect of general

anesthesia caused by:

- Volatile anesthetics \& opioids

- Prolonged fasting

- Pain

\section{- Mask Ventilation}

PONV affects $80 \%$ of high-risk surgical patients who undergo general anesthesia. Risk factors include

-Female gender

-Nonsmokers

-History of PONV

-Surgery within the past 24 hours

-Abdominal, ENT and gynecological surgery

PONV can cause life threatening post-op events such as wound dehiscence or disturbance, electrolyte disturbances, dehydration, pulmonary aspiration, hospital readmission, delayed discharge and increased healthcare costs.

\section{Methods}

- Eligibility Criteria

Peer-reviewed published studies

Must include opioid free anesthesia and opioid inclusive anesthesia

groups

Adult patient studies

ASA Score of III or lower

- Databases

Discovery Search Engine

AccessMedicine

UpToDate

- Data items

Agents used in place of opioids for experiment groups ncidence of postoperative nausea and vomiting (PONV) Use of antiemetics

Types of Studies

-Meta-analysis

Case control study

-Prospective cohort studies (4)

\section{Results}

The relevant charted data that was used for our results include pain scores, documented nausea and vomiting, as well as the use of opioids.

Specific Results:

-Reduction in PONV in OFA groups

-Reduction in rescue antiemetic use in OFA group (1 study)

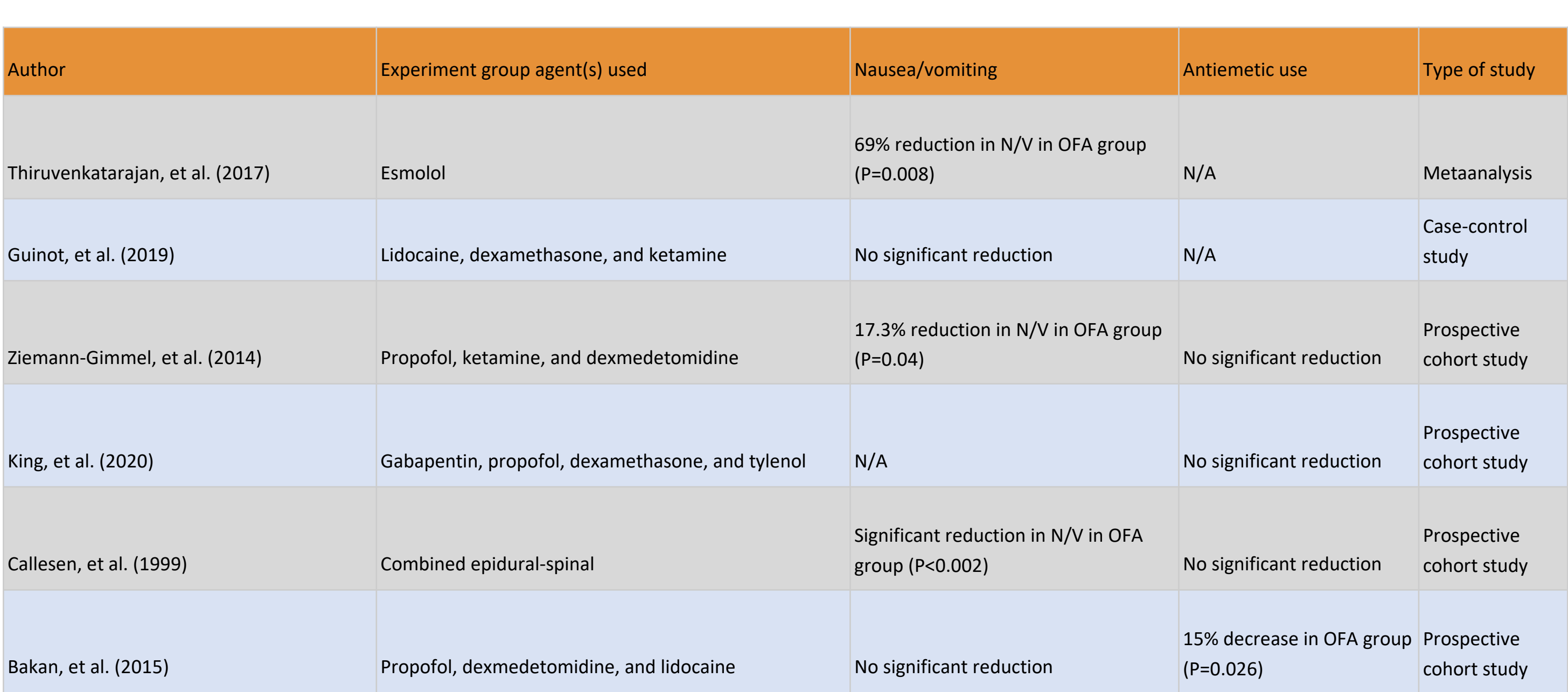

\section{Implications for Practice}

- Opioid alternatives are shown to reduce the incidence of post-operative nausea and vomiting

Esmolol, Ketamine, Propofol and Dexmedetomidine

- Education

-Ongoing professional development and education may be necessary to address hesitancy to change current anesthesia practice.

-The lack of professional education and hesitance to change current anesthetic practice from postoperative nausea and vomiting prevention with rescue medications with use of narcotics to opioid free anesthesia continues to be an issue.

- Lack of an Opioid-Free Anesthetic Algorithm Based on current best evidence, an opioid-free anesthesia algorithm may improve adherence to using opioid alternatives.

References

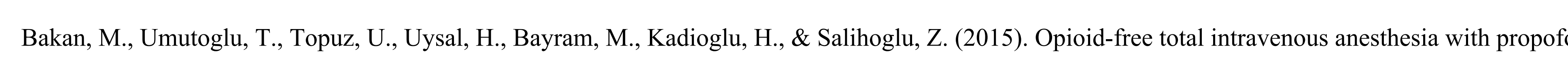

Anesthesiology (English Edition), 65(3).

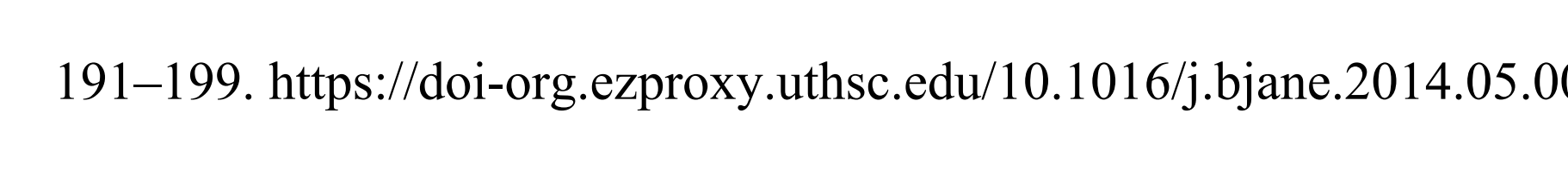

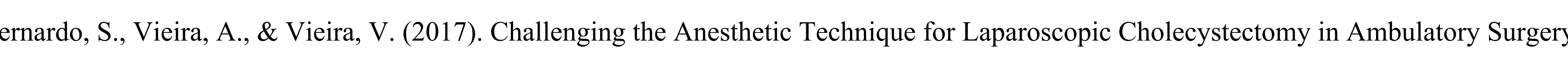

Ambulatory Surgery, 949 .

Retrieveref

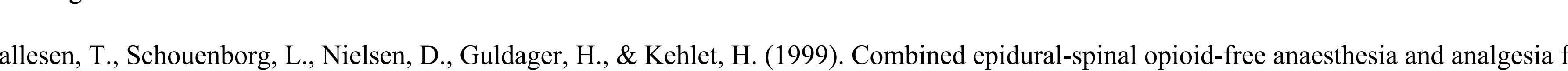

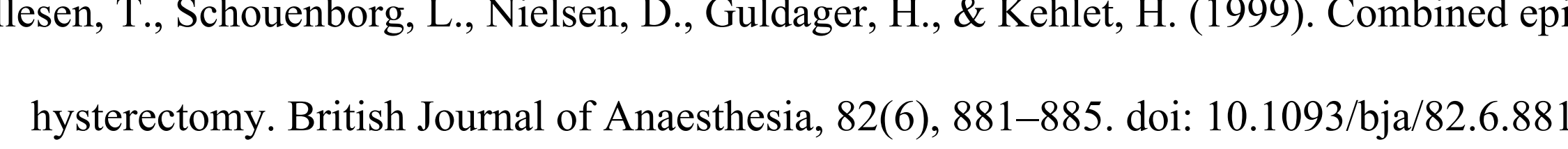

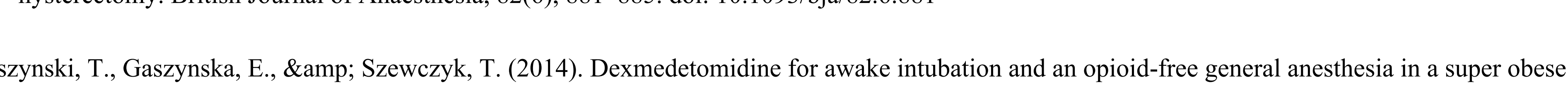

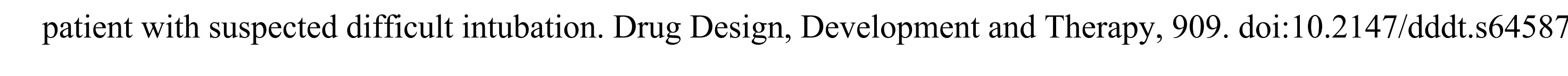

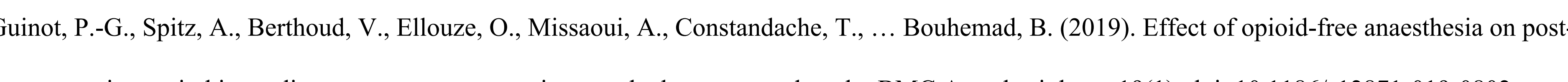

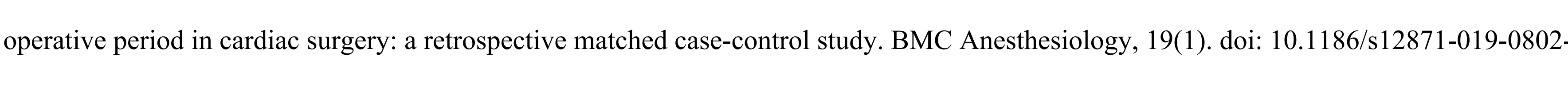

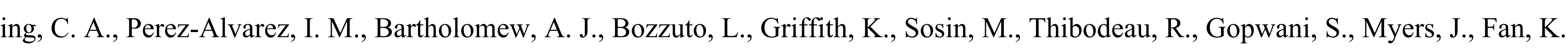

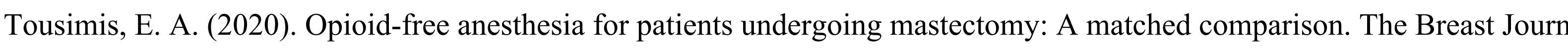

https://doi.org/10.1111/tbi.13999

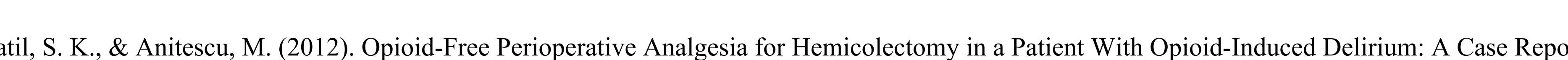

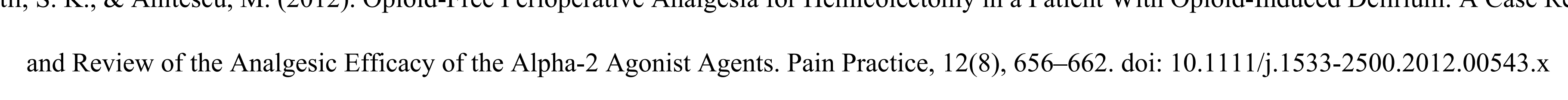

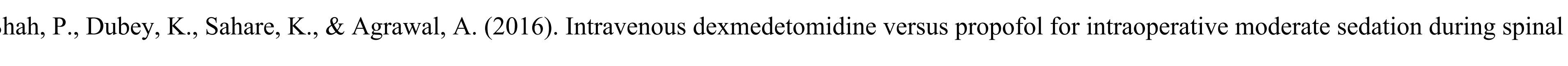

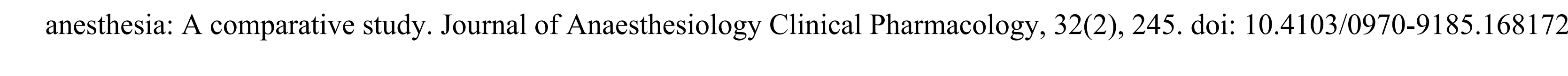

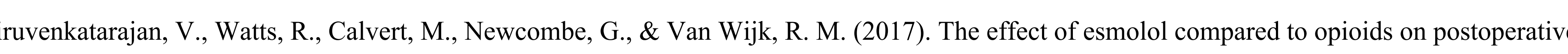

naussea and vomititg, postanesthesia 